\title{
Kernos
}

Revue internationale et pluridisciplinaire de religion grecque antique

15 | 2002

Varia

\section{Sabina MAZzoLDI, Cassandra, la vergine e l'indovina. Identità di un personaggio da Omero all'Ellenismo}

\section{Emilio Suárez de la Torre}

\section{OpenEdition \\ Journals}

\section{Édition électronique}

URL : http://journals.openedition.org/kernos/1431

DOI : 10.4000/kernos. 1431

ISSN : 2034-7871

\section{Éditeur}

Centre international d'étude de la religion grecque antique

Édition imprimée

Date de publication : 1 janvier 2002

ISSN : 0776-3824

\section{Référence électronique}

Emilio Suárez de la Torre, «Sabina mAzzoLdı, Cassandra, la vergine e l'indovina. Identità di un personaggio da Omero all'Ellenismo », Kernos [En ligne], 15 | 2002, mis en ligne le 22 avril 2011, consulté le 24 septembre 2020. URL : http://journals.openedition.org/kernos/1431 ; DOI : https:// doi.org/10.4000/kernos. 1431 
le livre, dont plusieurs index détaillés rendent la consultation particulièrement aisée.

Catherine Lecomte (Université de Liège)

Sabina Mazzoldi, Cassandra, la vergine e l'indovina. Identità di un personaggio da Omero all'Ellenismo, Pisa/Roma, Istituti editoriali e poligrafici internazionali, 2001. 1 vol. $14,5 \times 22,5 \mathrm{~cm}, 336$ p., 21 pl. ISBN : 888147-244-9.

Ces dernières années, le personnage de Cassandre, ainsi que le monde de la prophétie antique en général, ont connu un intérêt croissant, justifié par l'importance de cet aspect dans la religion et la littérature grecques. Le livre classique de J. Davreux (La légende de la prophétesse Cassandre d'après les textes et les monuments, Liège, 1942), encore utile, n'était pas du tout satisfaisant dans le traitement de certaines questions et, en ce qui concerne la partie iconographique, devait être mis à jour. Si le LIMC a contribué à remplir la dernière lacune, les études de D. Neblung (Die Gestalt der Kassandra in der antiken Literatur, Suttgart/Leipzig, 1997) et celle qui est maintenant le sujet de ce compte rendu ont satisfait géné reusement les exigences de révision des questions littéraires, et même (dans le cas de l'ouvrage de Mazzoldi) de l'étude des images.

Pour ceux qui pourraient se poser la question de la justification de cet ouvrage après la parution de celui de Neblung, je donne la parole à l'A. : «Scopo del lavoro non è fornire un repertorio delle fonti classiche (...) ma studiare le valenze del personaggio attraverso un approfondimento filologico e letterario di quelle testimonianze nelle quali la tipologia di Cassandra si definisce e si consolida, a partire da Omero fino alla letteratura ellenistica ». Il y a donc une différence d'extension chronologique et une autre, importante, dans l'analyse littéraire et linguistique des témoignages. Ce dernier trait est le plus remarquable du présent ouvrage et fait de lui un très utile instrument pour l'interprétation des textes étudiés (ce qui ne surprend pas, vu qu'il s'agit d'une thèse de doctorat dirigée par Paola A. Bernardini à Urbino).

Après la «Premessa » (p. 11-12) et l'Introduction (p. 13-21), le livre est divisé en deux parties. La première est une analyse approfondie du motif de la virginité

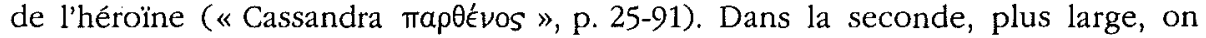
étudie les témoignages littéraires et les traits du langage prophétique qui caracté rise ces prédictions ( «Cassandra $\mu a ́ \nu T \iota s », p .93-283$ ). On ajoute un Appendice avec le lexique mantique (c'est-à-dire, «les mots les plụs significatifs employés par Cassandre ou en rapport avec elle » [p. 285, n. 1], p. 285-290). Suivent une excellente bibliographie (p. 291-318), un index des sources iconographiques, classé par thèmes (p. 321-330), un autre de noms mythiques et de notabilia (331334 ) et un dernier des passages discutés (p. 335-336). L'ouvrage se referme sur les photographies de 25 représentations figurées. L'édition est bien soignée et presque sans 'coquilles'.

La question de la virginité est analysée en trois chapitres : le premier a un caractère plus général, le deuxième traite le mythe du sacrilège d'Ajax et le troisième les rapports entre Cassandre et Agamemnon. Le premier ( Il yáfos mancato ", p. 27-30) établit bien que, depuis les sources les plus anciennes, le mariage de l'héroïne est un 'inaccomplissement' permanent. Elle est toujours 'projetée' vers ce but, mais elle n'y réussit jamais. En d'autre mots, Cassandre, en tant que femme, est définie négativement, presque comme une 'non-femme'. La lecture du deuxième chapitre ("Cassandra, Aiace e lo Góavov di Atena », p. 31-61) 
montre impeccablement qu'il y a deux phases dans le traitement du mythème, autant dans littérature que dans les représentations figurées. Dans la première, appartenant aux périodes archaïque et classique, c'est surtout la violation du droit d'asile qui est mise en relief, tandis que, peu à peu (jusqu'à devenir un motif dominant à la période hellénistique), c'est l'élément érotique, la violence sur la femme, qui prend le dessus. Or, je ne suis pas convaincu qu'une des raisons de cette évolution soit la perte de conscience de l'importance du droit d'asile (cf. p. 48). Les données épigraphiques, historiques et même les récits tardifs semblent contredire ce point de vue. Beaucoup plus importante me semble

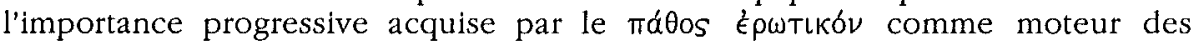
actions humaines, ce qui se trouve déjà partiellement chez Euripide et qui atteindra son point culminant, à nouveau, dans le roman de la période impériale. Dans ce même chapitre l'analyse des rites locriens en rapport avec Cassandre est très bien menée et très convaincante. La solidité des conclusions du chapitre précédent est confirmé par la coïncidence avec celles du troisième. Cassandre apparaît pour la première fois comme menace pour l'oikos dans l'Agamemnon d'Eschyle et c'est chez Euripide que le motif érotique l'emporte sur les autres : l'amour d'Agamemnon pour Cassandre devient un topos.

La seconde partie s'ouvre avec un chapitre sur le type de divination de

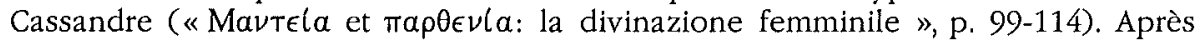
une délimitation précise des différences entre la Pythie, la Sibylle et Cassandre, ainsi que du rapprochement des deux dernières (confirmé par l'existence tardive d'une 'sibylle Cassandra'), on révise les deux traditions sur le mode d'acquisition des pouvoirs mantiques par Cassandre (octroyés par Apollon ou par le lèchement des oreilles par des serpents). Il s'agit d'une double possibilité bien connue dans les mythes des devins grecs qui, à mon avis, pourrait revéler un processus 'd'apollinisation' de ces traditions. Or, l'originalité du cas de Cassandre est précisément l'importance du motif érotique et le développement qu'il expérimente : encore une différence de 'genre' au niveau mythique.

«La $\mu \alpha \nu \tau \in l a$ di Cassandra nelle fonti letterarie e figurative », (p. 115-177) fait l'analyse des plus importants témoignages sur Cassandre, de l'épopée archaïque au théâtre du $\mathrm{V}^{\mathrm{e}} \mathrm{s}$. av. J. C. Le chapitre commence par des remarques sur le problème de la Cassandre homérique. Je partage pleinement l'opinion de l'A. sur l'absence de pouvoirs mantiques de cette héroïne chez Homère, ainsi que sur l'impossibilité de déduire des fragments du cycle quelle était la modalité de divination dans ces cuvres. Des pages sur la lyrique, il faut mettre en relief la qualité de l'analyse du Péan 8 a de Pindare, le premier témoignage du songe d'Hécube, qui est ici rappelé par Cassandre et interprété comme une annonce pour les Troyens au moment (paraît-il) du départ d'Alexandre/Pâris pour le voyage qui déclenchera la guerre. Le commentaire de l'A. est le plus complet dont nous disposons aujourd'hui, même après la parution de l'édition des Péans de Pindare d'I. Rutherford (Oxford, 2001), qui, d'ailleurs, propose de façon peu convaincante que ce poème provoqua la polémique entre Callimaque et Aristarque sur sa classification générique (péan/dithyrambe) et non celui de Bacchylide. Puis l'A. aborde le commentaire des fragments de la tragédie (elle laisse les grandes œuvres d'Eschyle et Euripide pour le chapitre suivant). De cet ensemble on doit souligner l'importance des conclusions sur l'Alexandre d'Euripide, à propos de la fonction du personnage et de son rôle dans l'ensemble de la pièce. Le chapitre se termine par des remarques sur les témoignages iconographiques.

Le troisième chapitre de cette partie (et dernier de l'ouvrage) est sans doute le plus original et remarquable ( Le profezie di Cassandra: modalità e lessico della 
divinazione », p. 179-283). L'Agamemnon d'Eschyle, les Troyennes d'Euripide et l'Alexandra de Lycophron, ainsi que des textes hellénistiques mineurs sont étudiés. Ces ouvres ont en commun la présentation des prophéties de Cassandre soumise à la transe mantique et deviennent des documents capitaux non seulement pour établir un profil précis de la modalité de la mantique représentée par l'héroïne à un moment donné, mais aussi pour mieux comprendre les conceptions grecques sur le procès divinatoire 'inspiré' et les traits des messages verbalisés qui en dérivent. Ces sont donc des textes assez particuliers, et dans la forme et dans le contenu, et des documents très représentatifs de leurs périodes respectives du point de vue social et littéraire. L'A. a réussit a délimiter parfaitement les traits complexes de la Cassandre d'Eschyle et de sa transe. Cette complexité est due à la variété de moments qui constituent la 'performance' de cette transe et de la différence d'intensité de chaque phase. La séquence sujet de cet analyse (vers 1072-1330, précédée d'un silence non moins expressif de Cassandre) est composée de quatre stades ( $c f$. le tableau de la p. 218): (A) des cris et invocation rituelle, (B) clairvoyance non médiate, (C) clairvoyance médiate et rationalité, et (D) prophétie rationnelle, intelligibilité. Chacun de ces stades peut être divisé en quatre phases en climax, d'extension variable. Cette analyse (présentée ici de façon trop résumée) est accompagnée d'une étude du langage qui caractérise la scène. En ce qui concerne les Troyennes, l'A. montre qu'il ne s'agit pas d'une transe, d'un 'invasamento del dio' (comme chez Eschyle), mais d'une modalité caractérisée, dans sa manifestation extérieure, par une mania définie explicitement ( $c f$. le lexique) comme bacchique et qui, par la modalité de son message, est plutôt un rapport qu'une prophétie en tant que telle. Cette 'folie' contribue à renforcer la marginalité du personnage et se trouve à la base du traitement de Cassandre dans la littérature européenne postérieure.

Je soulignerai enfin la finesse et la rigueur méthodologique du commentaire de l'Alexandra de Lycophron. Ce texte, profondément représentatif des traits de la littérature hllénistique, transforme la prophétie de Cassandre (moyennant une rupture entre elle et le destinataire, en ayant recours au gardian de la prison, qui en fait le rapport) en une énigme prolongée. "L'alvi $\gamma \mu \alpha$ è predilezione alessandrina nell'ambito del clima culturale e intellettuale in cui visse Licofrone: l'autore gli conferisce carattere normativo all'interno del monologo oracolare, piegando la profezia ai dettami della poetica vigente, e tramite l'uno legittima l'altra " (p. 259). On soulignera aussi l'utilité du tableau des p. 267-269, qui contient un schéma de l'Alexandra où les niveaux chronologiques du poème (temps présent, 'flashback', temps futur et prophétie sur un futur qui précède les événements du récit principal) sont typographiquement différenciés.

Il s'agit donc d'une importante contribution à la connaissance de la prophétie dans le Monde Ancien, à l'approfondissement de l'étude des figures mantiques du mythe et à l'analyse littéraire et linguistique des ouvres qui accueillent le personnage.

Emilio Suárez de la Torre (Universidad de Valladolid)

Pierre Brulé, Les femmes grecques à l'époque classique, Paris, Hachette Littératures, 2001. 1 vol. $14 \times 22 \mathrm{~cm}, 282$ p. (Coll. La vie quotidienne). ISBN : 2-01-235480-7.

Avec un tel interprète, la femme grecque ne pouvait rester muette, fade et recluse. En effet, après son imposante thèse sur La fille d'Athènes (Paris, 1987), P. Brulé était tout désigné pour dessiner ce portrait féminin, en laissant de côté ce que l'exercice de la thèse peut avoir de contraignant. Le propos est donc alerte, le 